\title{
Recent Development in Phosphonic Acid-Based Organic Coatings on Aluminum
}

\author{
Ruohan Zhao, Patrick Rupper * (i) and Sabyasachi Gaan * \\ Additives and Chemistry Group, Advanced Fibers, Empa, Swiss Federal Laboratories for Materials Science and \\ Technology, Lerchenfeldstrasse 5, 9014 St. Gallen, Switzerland; ruohan.zhao@empa.ch \\ * Correspondence: patrick.rupper@empa.ch (P.R.); sabyasachi.gaan@empa.ch (S.G.); \\ Tel.: +41-587-657-559 (P.R.); +41-587-657-611 (S.G.)
}

Received: 11 July 2017; Accepted: 21 August 2017; Published: 23 August 2017

\begin{abstract}
Research on corrosion protection of aluminum has intensified over the past decades due to environmental concerns regarding chromate-based conversion coatings and also the higher material performance requirements in automotive and aviation industries. Phosphonic acid-based organic and organic-inorganic coatings are increasingly investigated as potential replacements of toxic and inefficient surface treatments for aluminum. In this review, we have briefly summarized recent work (since 2000) on pretreatments or coatings based on various phosphonic acids for aluminum and its alloys. Surface characterization methods, the mechanism of bonding of phosphonic acids to aluminum surface, methods for accessing the corrosion behavior of the treated aluminum, and applications have been discussed. There is a clear trend to develop multifunctional phosphonic acids and to produce hybrid organic-inorganic coatings. In most cases, the phosphonic acids are either assembled as a monolayer on the aluminum or incorporated in a coating matrix on top of aluminum, which is either organic or organic-inorganic in nature. Increased corrosion protection has often been observed. However, much work is still needed in terms of their ecological impact and adaptation to the industrially-feasible process for possible commercial exploitation.
\end{abstract}

Keywords: phosphonic acids; corrosion protection; aluminum; sol-gel; coatings

\section{Introduction}

Aluminum ( $\mathrm{Al})$ and its alloys have been widely used in engineering applications because of their higher strength to weight ratio, ductility, formability, and lower costs. In many applications, such as in aircraft, automobiles, and structural parts in buildings, its corrosion resistance becomes very important. Aluminum, by itself, is relatively stable to corrosion because it readily oxidizes to form a passive protective oxide layer on its surface which is robust and does not simply flake off. However, the galvanic corrosion of aluminum is very critical and is complicated in various alloys and under acidic $\mathrm{pH}$. An excellent review on corrosion protection of aluminum prior to the year 2000 can be found in the literature [1]. The need to develop chromate-free treatments for aluminum is becoming increasingly important. Many organic and inorganic protective coatings are being investigated by researchers with the potential advantages and shortcomings [1]. Organic-inorganic conversion coatings are quite attractive due to the simplicity of their application and possible favorable interaction with organic layers in hybrid materials, which offer enhanced corrosion resistance and mechanical performance [2].

A variety of phosphonic acids is commonly used to modify the surfaces of metals and their oxides for their corrosion protection, stabilization as nano-particles, adhesion improvement to organic layers, hydrophobization, hydrophilization, etc. They are excellent chelating agents and bind very strongly to metals resulting in different 1D to 3D metal organic frameworks (MOFs), also called metal phosphonates $[3,4]$. They form hydrolytically-stable bonds with the metals and provide excellent 
coverage compared to many thiols, silanes, and carboxylic acids. A phosphonate corrosion inhibitor adsorbs well on the metal surface, reducing its solubility in aqueous media and, thus, decreases the area of active metal surface and increases the activation energy to hydrolysis. Such phosphonic acids can be a simple molecule where the phosphonic acid is attached to an alkyl or aromatic group. Self-assembly of such phosphonic acids on aluminum surface is well studied where the binding of these molecules to aluminum can be monodentate, bidentate, or tridentate [5-7]. More recently there is an increased impetus to develop functional phosphonic acids, which can not only bind to the metal surface but also provide linkage to an organic matrix in multicomponent systems $[8,9]$. In some cases, phosphonic acid acts as a linker between aluminum and a hydrophobic polymerizable group (pyrrole) [10] or chemically-stable protective material (graphene oxide) [11]. Such molecules are applied on the surface of aluminum via dip coatings, spray from aqueous solutions, or are incorporated in a polymeric matrix (adhesives, glues, or paints) which is then coated on the metal surface. A novel ultrasonic assisted deposition (USAD) method has also been developed to coat phosphate films on aluminum [12]. This application procedure is believed to improve the interaction of the phosphonic acid to the aluminum surface.

Functional phosphonic acids with hydrophobic aliphatic groups or fluorinated groups can increase the hydrophobicity of the metal surface, thereby acting as barrier to aqueous solutions and improving its corrosion protection. In some cases the functional groups of phosphonic acids react with organic layers (adhesives) and provide a stable barrier against aqueous environments.

The following sections first briefly summarize the methods for characterization of phosphonic acids on aluminum surfaces and several techniques used to evaluate the corrosion behavior, in order to understand the following chapter, which deals with the application of various types of phosphonic acids on aluminum, together with their physico-chemical behavior relevant to corrosion protection and adhesion to organic layers. Thereby, a distinction has been made between the aluminum surface treatment with phosphonic acids or phosphates as a pre-coating and surface coatings dissolved in paints that contain phosphonic acids.

\section{Characterization of the Phosphonic Acid-Modified Aluminum Surfaces}

Self-assembled monolayers (SAM) are ordered molecular assemblies and are commonly used to modify aluminum surfaces. They are spontaneously formed by the adsorption of molecules with head groups that show affinity to a specific substrate [13]. Phosphonic acids form SAMs on aluminum surfaces by condensation reactions of the acid functional group with basic surface-bound alumino-hydroxyl species. Thereby, the phosphonic acid head group shows a strong affinity to the aluminum substrate, creating an alumino-phosphonate linkage via a strong Al-O-P chemical bond:

$$
\mathrm{R}-\mathrm{PO}(\mathrm{OH})_{2}+\mathrm{Al}-\mathrm{OH} \rightarrow \mathrm{R}-(\mathrm{OH}) \mathrm{OP}-\mathrm{O}-\mathrm{Al}+\mathrm{H}_{2} \mathrm{O} \text { [5-7] }
$$

To investigate a successful formation of the organophosphorus layers, including information about the properties of the films (surface morphology, structural ordering, density and uniformity, binding mode to the aluminum substrate, as well as stability), different analytical methods have been applied in the literature. A detailed characterization of the layers is necessary to understand their intended function (for instance corrosion protection, adhesion promotion, (non)-wettability, surface passivation). Table 1 summarizes the various analytical techniques that have been used in the last 20 years, or so, to study the organization and chemistry of organophosphorus compounds on an aluminum substrate. Various characterization techniques have been categorized by us into three groups: Surface morphology; presence, composition, and stability of layers; orientation of molecules and binding mode to aluminum, and are all briefly explained in the remainder of this section. 
Table 1. Commonly-employed surface characterization techniques.

\begin{tabular}{ccc}
\hline Characterization & Technique & Literature \\
\hline Surface morphology & Scanning electron microscopy (SEM) & {$[9,14,15]$} \\
& Atomic force microscopy (AFM) & {$[8,15]$} \\
\hline & X-ray photoelectron spectroscopy (XPS) & {$[6-8,11,16-21]$} \\
Presence, composition & Auger electron spectroscopy (AES) & {$[22]$} \\
and stability of layer & Fourier-transform infrared spectroscopy (FTIR) & {$[8,11,14,16,17,23,24]$} \\
& Time-of-flight secondary ion mass spectrometry (ToF-SIMS) & {$[6]$} \\
\hline \multirow{2}{*}{ Orientation of molecules } & Water contact angle measurements (WCA) & {$[5,7,10,16,25,26]$} \\
and binding mode to & Anfrared reflection absorption spectroscopy at grazing angle (IRRAS) & {$[5,12,16,29-33]$} \\
aluminum & X-ray photoelectron spectroscopy (XPS) & {$[20,28,34]$} \\
& Solid-state ${ }^{31}$ P nuclear magnetic resonance (NMR) & {$[12,31-33]$} \\
\hline
\end{tabular}

\subsection{Surface Morphology}

Microscopy measurements, i.e., optical microscopy, scanning electron microscopy (SEM) and atomic force microscopy (AFM), were used to investigate the surface morphology of the organophosphorus self-assembled monolayers onto the aluminum substrate. Physical characteristics, like uniformity and homogeneity of the surface phosphatization, were obtained by comparing the coated with the bare aluminum substrate using SEM [14]. AFM has been used to obtain information about the surface smoothness $[8,15]$.

\subsection{Presence, Composition, and Stability of Layer}

A variety of surface sensitive analytical techniques has been used to investigate the presence of the layer and its composition. X-ray photoelectron spectroscopy (XPS) and Auger electron spectroscopy (AES) are both methods which determine the elemental composition in the first couple of nanometers of a surface and, hence, were often applied to verify the presence of phosphorus, indicating a successful organophosphorus coating chemically-bound to aluminum $[6,8,11,16,17,19-22]$. As a general preparative step, the samples were usually rinsed/washed after the coating process to remove the residual chemicals and physically adsorbed organophosphorus compounds. In addition to the determination of the presence of the organophosphorus coating, XPS was also used to study the concentration of the organic molecules on the aluminum surface [18]. The experimental phosphorus to aluminum concentration ratio allowed the quantification of the organophosphorus molecule surface concentration and modelling of the XPS data helped to determine the thickness of the monolayer [18]. Thereby, the ratio of signals from the coating and from the underlying aluminum substrate was recorded, from which the coating thickness can be derived. Depth profiling via argon ion sputtering in combination with XPS was also used to determine the coating thickness (especially for thicker layers). The comparison between experimental and theoretical values for the carbon-to-phosphorus ratio was used as proof that chemically-uniform organophosphorus films formed on aluminum [7]. The recording of characteristic binding energies allowed to follow the individual steps in the decomposition of the phosphonates on the aluminum surface [20]. Table 2 summarizes XPS binding energies found in the literature for organophosphorus layers on metal surfaces.

Another analytical technique often used to study the presence and composition of organophosphorus layers on aluminum is reflectance Fourier-transform infrared spectroscopy (FTIR), often measured at a grazing angle to increase the surface sensitivity. FTIR was applied to investigate the adsorption of the coating by detecting specific vibrational modes involving phosphorus bonds $[8,11,14,16,17,23,24]$. Table 3 contains a summary of frequencies of vibrational modes involving the phosphorus atom found in the literature for self-assembled organophosphorus monolayers on aluminum. 
Table 2. XPS binding energies relevant to phosphonic acids modified aluminum surface.

\begin{tabular}{ccc}
\hline Assignment & Binding Energy (eV) & Literature \\
\hline$A l 2 p$ & & {$[7,16,20,35,36]$} \\
\hline metal & $72.3-73.3$ & \\
Al-oxide, Al-hydroxide & $74.2-76.2$ & \\
Al-phosphonate & $\sim 75.5$ & {$[16,34,37]$} \\
\hline$P 2 p$ & & \\
\hline phosphonates & $133.3-134.2$ & \\
\hline$P$ 2s & & \\
\hline P-C, P=O, P-O & $192.5-192.7$ & \\
\hline O 1s & & \\
Al-oxide & $530.6-531.1$ & \\
Al-hydroxide & $532.3-532.4$ & \\
water & $534-535$ & \\
P=O, P-O ${ }^{-}$, P-O-Metal & $531.4-532.1$ & \\
P-OH, P-OR & $532.6-534.3$ & \\
\hline
\end{tabular}

Table 3. Infrared vibrational modes involving the phosphorus atom for the self-assembled organophosphorus layer on aluminum.

\begin{tabular}{ccc}
\hline Assignment & Peak Position $\left(\mathbf{c m}^{-\mathbf{1}}\right)$ & Literature \\
\hline $\mathrm{P}-\mathrm{O}$ sym. stretch & $910-960$ & {$[5,24,27,30,37]$} \\
$\mathrm{P}-\mathrm{O}$ asym. stretch & $1000-1040$ & {$[8,11,12,16,24,27,30-32,37]$} \\
\hline $\mathrm{P}=\mathrm{O}$ stretch & $1100-1250$ & {$[37]$} \\
\hline $\mathrm{PO}_{2}{ }^{-}$sym. stretch & $1000-1070$ & \\
$\mathrm{PO}_{2}{ }^{-}$asym. stretch & 1160 & {$[5,8,24,29,37]$} \\
$\mathrm{PO}_{3}{ }^{2-}$ sym. stretch & $960-1060$ & {$[16,27,31]$} \\
$\mathrm{PO}_{3}{ }^{2-}$ asym. stretch & $1115-1140$ & $2500-2750$ \\
$\mathrm{P}-\underline{\mathrm{OH}}$ stretch & &
\end{tabular}

Time-of-flight secondary ion mass spectrometry (ToF-SIMS), as a complementary technique to XPS, has also been used to demonstrate the presence of the organophosphorus layer, thereby investigating selective functionalization on aluminum and glass regions of a surface by mapping analysis [6]. In addition, surface plasmon resonance spectroscopy (SPR) was applied to examine the kinetics of the adsorption process [27]. Thereby, it was found that the adsorption process of phosphonic acids on aluminum starts very quickly and reaches a plateau after some minutes.

Water contact angle (WCA) measurements were used to investigate the hydrophilicity and hydrophobicity of the surface. A change compared to a reference untreated aluminum surface indicated the successful formation of an organophosphorus layer. In addition, WCA measurements were also applied to study the orientation of the molecules (see also below) because in well-ordered monolayers, the access of the water drop to the aluminum surface is reduced [7]. From the investigation of the temporal behavior of WCA, information about surface coverage and chain ordering was obtained [5]. Finally, the stability of the adsorbed layers was determined by recording advancing and receding contact angles by repeated cycling $[10,26]$.

\subsection{Orientation of Molecules and Binding Mode to Aluminum}

In order to characterize the binding (orientation and mode) of the organophosphorus molecules to an aluminum surface, mostly three methods have been used in the literature: Infrared reflection absorption spectroscopy (IRRAS) at grazing angle (often applying polarized infrared radiation), X-ray photoelectron spectroscopy (XPS) at a fixed electron take-off angle as well as angle-resolved (ARXPS) and solid-state ${ }^{31} \mathrm{P}$ nuclear magnetic resonance (NMR) spectroscopy. 
The most expected orientation with the phosphonic acid group reacting with the surface hydroxyl groups of the aluminum substrate and the hydrocarbon tail and/or terminal functional groups on top was proven by angle-resolved XPS. The carbon (from the tail) to phosphorus (from the head group) intensity ratio was determined at low and high electron take-off angles, representing two different information depths $[7,10,27,28]$. In addition, using infrared reflection absorption spectroscopy (IRRAS) and comparing the intensities of vibrations polarized perpendicular or parallel to the hydrocarbon backbone, information about the orientation of the organophosphorus molecule was also obtained [5].

The phosphonic group can bind to the aluminum atom via a direct $\mathrm{P}-\mathrm{O}-\mathrm{Al}$ bond in different modes, i.e., mono-, bi-, or tridentate, depending on whether one, two or all three oxygen atoms from the phosphonic group are involved, respectively. It has been found that both the deposition mode (for instance stirring, sonication), as well as the structure of the phosphonates, play a role [12]. Additionally, the evolution from tridentate to lower binding modes as film formation proceeds (the increase of coverage can change the chain ordering) was observed in the literature [5]. These behaviors might explain the variety of binding modes reported in the literature for organophosphorus layers on aluminum.

Table 3 summarizes the frequencies of infrared vibrational modes found in the literature for self-assembled organophosphorus layers on aluminum. By observing which vibrational bands are present and which are not, certain binding modes have been assigned (mostly bidentate $[16,29]$ and tridentate $[12,32,33]$, and some monodentate [30]). For instance, a missing $\mathrm{P}-\mathrm{OH}$ stretching mode together with the presence of $\mathrm{PO}_{3}{ }^{2-}$ stretching modes (deprotonation of the phosphonic acid group) in the IR spectrum indicates the formation of a bidentate binding. Mixtures of binding modes have also been found $[5,30,31]$.

As a second technique to investigate the binding mode of organophosphorus molecules to aluminum, X-ray photoelectron spectroscopy (XPS) was used. Table 2 summarizes the XPS binding energies found in the literature for self-assembled organophosphorus layers on aluminum. As can be seen from Table 2, due to overlapping bands, neither the aluminum nor the phosphorus signal allows to clearly differentiate between various binding modes of phosphorus to aluminum. It has to be noted that a trend in the experimental binding energy to increase for the structure $\left[\mathrm{PO}_{n}(\mathrm{OR})_{m}\right]^{y-}$ is observed as the ratio of $n: m$ of "free" O ligands $(n)$ to covalently bound OR ligands $(m)$ is stepwise changed from 4:0 to 3:1 to 2:2 and to 1:3. Therefore, also the XPS phosphorus binding energy has been used to assign the binding mode (i.e., mono-, bi-, or tridentate) [34]. However, a final conclusion cannot be drawn just from the P $2 p$ signal and further experimental evidence is needed. The $\mathrm{O} 1 \mathrm{~s}$ signal is of particular interest regarding the assessment of the type of bond between organophosphorus and the aluminum substrate. As the binding energy for $\mathrm{P}=\mathrm{O}$ and $\mathrm{P}-\mathrm{O}-\mathrm{Metal}$ is different from the one for $\mathrm{P}-\mathrm{OH}$ and $\mathrm{P}-\mathrm{OR}$ (see Table 2), the ratio of the intensities at these two binding energies was used to determine the binding mode. In agreement with other studies using IR (see above), a mixed monodentate and bidentate mode was found [34]. As a third method, the linewidth and shifts of peaks in ${ }^{31} \mathrm{P}$ NMR spectra have been used to study the bonds between the aluminum and the oxygen atoms of the organophosphorus molecule and to assign a specific binding mode by comparison with literature data [12,31].

\section{Corrosion Evaluation Methods}

The corrosion resistance of the coated aluminum surface is evaluated mainly by four different ways: Electrochemical measurements, conventional measurements, spectral analysis and surface analysis, and are briefly summarized in Table 4 . In most cases treated aluminum substrates are coated with an epoxy layer to study its adhesion and corrosion performance. The corrosion resistance of aluminum substrates is highly related to the microstructure, chemical composition, electrochemical behavior of the coating, and to the interface between coating/metal. Therefore, the measurements mentioned in Table 4 are mostly comprehensive and are used to gather more information in order to analyze the corrosion behavior from different perspectives. 
Table 4. Common methods for corrosion measurements.

\begin{tabular}{ccc}
\hline Measurements & Examples & Literature \\
\hline $\begin{array}{c}\text { Electrochemical } \\
\text { measurements }\end{array}$ & $\begin{array}{c}\text { Electrochemical noise method (ENM) } \\
\text { Potentiodynamic polarization measurements } \\
\text { Electrochemical impedance spectroscopy (EIS) }\end{array}$ & {$[22]$} \\
\hline $\begin{array}{c}\text { Localized electrochemical } \\
\text { techniques }\end{array}$ & $\begin{array}{c}\text { Scanning Kelvin Probe (SKP) and Scanning } \\
\text { Vibrating Electrode Technique (SVET) }\end{array}$ & {$[42]$} \\
\hline Conventional & Immersion test & {$[14,23,41]$} \\
measurements & Salt spray test & {$[8,27]$} \\
Spectral analysis & Weight loss treatment & {$[43]$} \\
\hline Wettability & Infrared spectroscopy (IR) & {$[12]$} \\
\hline
\end{tabular}

\subsection{Electrochemical Measurements}

Electrochemical changes occurring during the coating failure can be detected via an electrical signal which could provide information of metal corrosion and coating property alterations. Quantitative and semi-quantitative evaluation of coatings could be achieved by electrochemical measurements, such as EIS, ENM, and hydrogen permeation current method.

\subsubsection{Electrochemical Impedance Spectroscopy (EIS)}

EIS is a non-destructive measurement, which can provide time dependent surface information in a corrosive medium. A small amplitude sinusoidal alternating signal was added in the coating/primer/metal system. Through analyzing the impedance spectrum and the admittance spectrum, an equivalent circuit model, shown in Figure 1, was built to evaluate electrochemical information of the coating system. During the electrolyte penetration through the coating, $C_{\text {coat }}$ increases and $R_{\text {coat }}$ decreases. It is confirmed that a surface with an impedance at the low frequency below $10^{7} \Omega \cdot \mathrm{cm}^{2}$ is considered a poor protective barrier [44].

EIS is a nondestructive method and provides fast detection and comprehensive information on coating corrosive behavior. The G106-89 standard has been developed for EIS measurement. However, EIS only provides general information on corrosion behavior of the surface, which means it cannot help understand the mechanism of the corrosion start point [45].

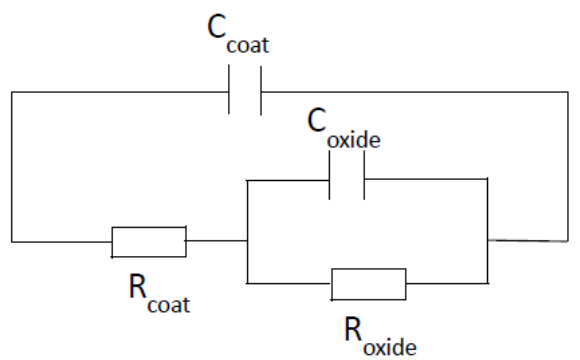

Figure 1. An equivalent circuit model for epoxy coated aluminum. $R_{\text {oxide }}$ is the resistance of oxide aluminum layer, $C_{\text {oxide }}$ is the capacitance of oxide aluminum layer, $R_{\text {coat }}$ is the resistance of the coating, and $C_{\text {coat }}$ is the capacitance of the coating. (Reproduced from [41] with permission; Copyright 2004 Elsevier).

\subsubsection{Electrochemical Noise Method (ENM)}

Electrochemical noise can be described as naturally-occurring fluctuations in potential and current around a mean value in the electrochemical cell [46]. The parameters voltage noise $\left(\sigma_{v}\right)$ and current 
noise $\left(\sigma_{i}\right)$ can be derived from the fluctuations. The noise resistance $R_{n}$, which can be calculated by the Ohms Law, is used to evaluate the corrosion resistance (see Equation (1)):

$$
R_{n}=\frac{\sigma_{v}}{\sigma_{i}}
$$

The higher the value of $R_{n}$ is, the better the corrosion resistance of the coating [22]. When the value of noise resistance $R_{n}$ is less than $10^{6} \Omega \cdot \mathrm{cm}^{2}$, the coating shows poor corrosion resistance. When $R_{n}$ is more than $10^{8} \Omega \cdot \mathrm{cm}^{2}$, the coating exhibits good corrosion protection. The value between $10^{6}$ and $10^{8} \Omega \cdot \mathrm{cm}^{2}$ indicates an intermediate level of corrosion resistance [47].

ENM is an electrically non-intrusive sensitive method, which requires only a few minutes for a single measurement [48]. Recently, it has become the main measurement method for determining the metal corrosion rate, the localized corrosion process, and is widely used in industry for corrosion detection.

\subsubsection{Potentiodynamic Polarization Measurements}

Potentiodynamic anodic polarization can characterize the metal corrosion behavior by its current potential relationship. It can be used to determine the function and the type of inhibitor. The sample potential is scanned slowly in the positive direction, which means it forms an oxide coating during the test. The passivation tendencies and inhibitors influence can be easily studied by this method. Meanwhile, the corrosion behavior of different coatings or metals can be compared on a rational basis, and it can be used as a pretest to give a suitable corrosive condition range for further long-term measurements. The general corrosion behavior of coated substrates can be evaluated by the corrosion potential $E_{\text {corr }}$. Specifically, the more positive $E_{\text {corr }}$ and the lower the current values, the better the corrosion resistance [22].

\subsubsection{Localized Electrochemical Techniques}

For many corrosion phenomena, high resolution instead of average data about the behavior of an electrochemically-active surface is required (for instance to differentiate small local anodes and cathodes on a metal surface) [42]. A scanning Kelvin probe (SKP), as a non-contact and non-destructive method, allows measuring and mapping the local potential and current difference on the microscale. The scanning vibrating electrode technique (SVET) enables to spatially characterize corrosion activity by measuring the potential gradients in the electrolyte due the presence of anodic and cathodic areas on a metal surface. These localized techniques find applications in different corrosion investigations like effect of microstructure and finish on corrosion initiation, localized corrosion phenomena, and detection of electrochemically-active pin-hole defects in coatings.

\subsection{Conventional Measurements}

Conventional corrosion measurement methods are inexpensive and easy to perform. Different national and international standards have been developed which include immersion, salt spray, damp heat, gas corrosion, and filiform corrosion tests. They are commonly used in industry. However, they are not suitable for the corrosion kinetics study or the corrosion mechanism study as they provide qualitative results, usually involving long test cycles and poor repeatability.

\subsubsection{Immersion Test}

The coated substrates are directly immersed in the corrosive medium. After a certain exposure to corrosion medium, the damage or corrosion of the coating is observed to evaluate corrosion resistance. The immersion tests are widely used for different coatings in different corrosion media. The common immersion tests are water resistance test, salt water resistance test, acid resistance test, and various organic solvent resistance tests. One can find numerous national immersion tests specific to a country. Some international ASTM standards for immersion tests are B895-05, G44, and G110. 


\subsubsection{Salt Spray Test}

The salt spray test is the most classic and widely-used method to evaluate the corrosion resistance of coatings. The first international salt spray standard, ASTM B117, was recognized in 1939. The coated substrates are scratched first and then the test is carried out in a closed test chamber, where salt water ( $5 \% \mathrm{NaCl}$ solution, $\mathrm{pH} 6.5-7.2$ ) is sprayed using pressurized air at $35 \pm 2{ }^{\circ} \mathrm{C}$. Later standards include ISO9227, JIS Z 2371, and ASTM G85. To modify the corrosion condition, acetic acid-salt spray (ASS), copper-accelerated acetic acid-salt spray (CASS), prohesion cycle test as well as various modified versions were established.

\subsection{Spectral Analysis}

The decomposition of a polymer in the coating is one of the main reason that causes coating failures and metal corrosion, which would lead to further deposition. The chemical changes of the polymer and the inorganic corrosion products can be quantitatively detected by, for example, FTIR, infrared microscopy, or laser Raman spectroscopy (LRS). They can be used to study the corrosion performance and corrosion mechanism because of the highly-precise positioning and quantification [45]. The detail about spectral analysis has been described in Sections 2.2 and 2.3.

\subsection{Surface Analysis}

The various properties of the coating are highly related to its microstructure, chemical composition, and its bonding condition at the interface of coating/metal. The surface changes during the corrosion process were characterized using the same analytical methods as already described in Section 2.1.

\section{Aluminum Surface Treatments with Phosphonic Acids}

The following sections describe the various types of phosphonic acids and some phosphates which have been used to modify the surface of aluminum with the main objective to improve its corrosion protection and/or adhesion promotion to organic layers. A list of phosphonic acids commonly used to improve corrosion protection of aluminum is shown in Table 5. Some of these phosphonic acids have simple structures (PPA or MSAP), others are more functional phosphonic acids with terminal groups like vinyl (VPA), amino (APP), and pyrrol (Cn-Ph-P).

Table 5. Chemical name, structure, and abbreviation of organophosphonic acids.

\begin{tabular}{cccc}
\hline Chemical Name & Abbreviation & Literature \\
\hline Phenylphosphonic acid & PPA & [14,22] \\
1,12-dodecyldiphosphonic acid & VPA & [49] & DDP \\
\hline Amino trimethylene phosphonic acid & ATMP & [15] \\
\hline
\end{tabular}


Table 5. Cont.

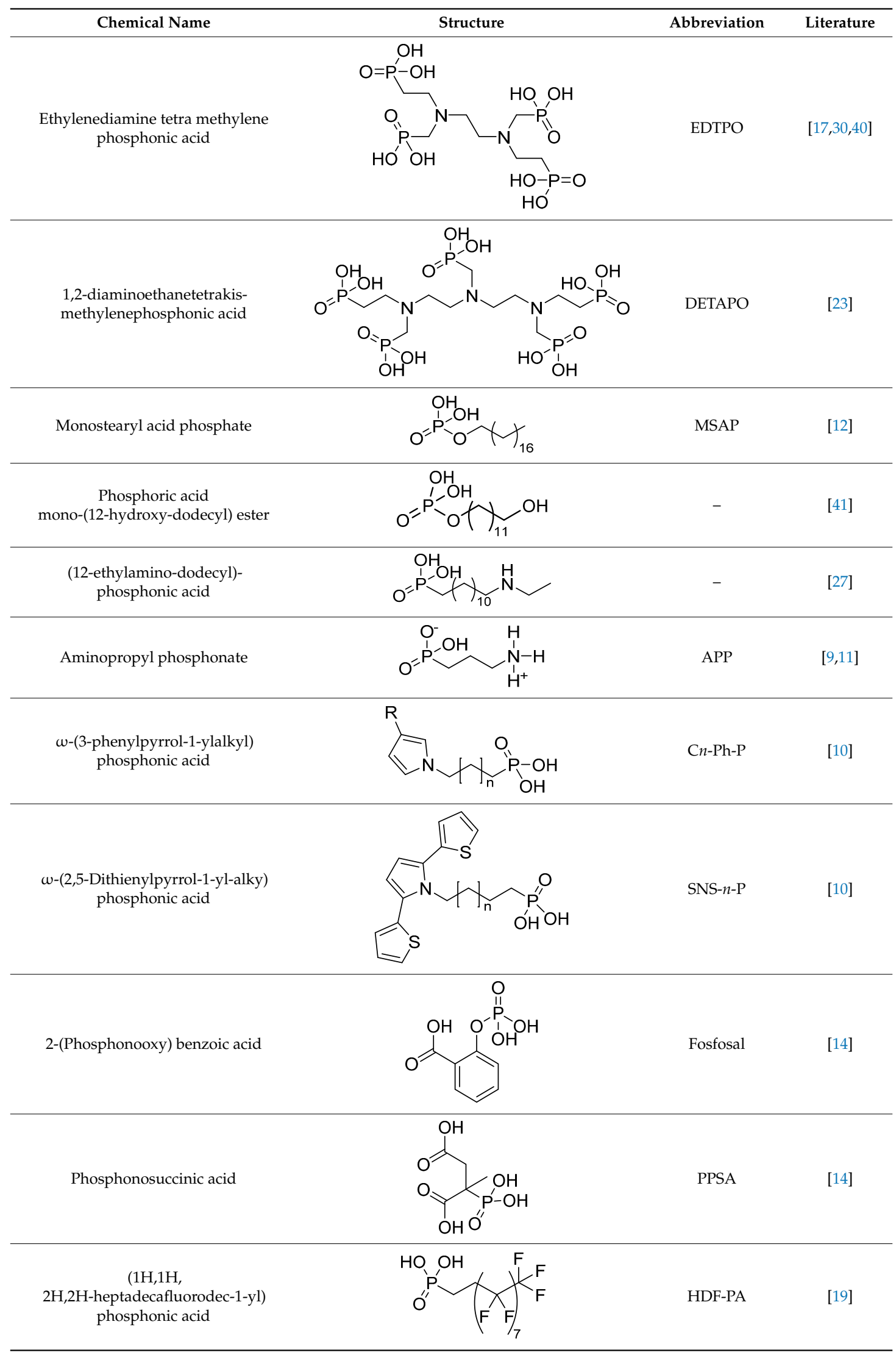


Table 5. Cont.

\begin{tabular}{|c|c|c|c|}
\hline Chemical Name & Structure & Abbreviation & Literature \\
\hline Fluoro alkyl phosphates & $\begin{array}{c}R_{x} \mathrm{PO}_{2} \\
R=\mathrm{CF}_{3}\left(\mathrm{CF}_{2} \mathrm{CF}_{2}\right)_{z}\left(\mathrm{CH}_{2} \mathrm{CH}_{2} \mathrm{O}\right) \\
x=1,2 \text { or } 3, x+y=3, z=1 \text { to } 7\end{array}$ & Zonyl UR & [12] \\
\hline Poly (vinyl phosphonic acid) & $\mathrm{HO}-\stackrel{\mathrm{O}}{\mathrm{P}}-\mathrm{OH}$ & PvPA & [24] \\
\hline
\end{tabular}

\subsection{Phosphonic Acids Used as Pre-Coating}

\subsubsection{Phenylphosphonic Acid (PPA)}

In an earlier work, it was found that a hydrophobic sol-gel film can provide good corrosion resistance to aluminum [50]. Subsequently, the same researchers incorporated organic phenyl phosphonic acid as an anion in a hydrophobic sol-gel film to further enhance the protection of aluminum against pitting corrosion. It was expected that such combinations of phosphonic acid and sol-gel coatings would be beneficial in corrosion protection. Various sol-gel coatings with two different anions were coated on the aluminum substrate. The formulations of such coatings used in this research are shown in Table 6.

Table 6. Various formulations of sol-gel coatings.

\begin{tabular}{cccccccc}
\hline $\begin{array}{c}\text { Sol-Gel } \\
\text { Chemistry }\end{array}$ & PTMOS & PTMOS & $\begin{array}{c}\text { PTMOS }+ \\
\text { TMA }\end{array}$ & PTMOS & TEOS & TEOS & $\begin{array}{c}\text { TEOS + } \\
\text { TMA }\end{array}$ \\
\hline Anion & - & PPA & PPA & TBPA & - & PPA & PPA \\
\hline
\end{tabular}

Phenyltrimethoxysilane (PTMOS), tetraethyl orthosilicate (TEOS), $N$-trimethoxysilylpropyl- $N, N, N$-trimethyl ammonium chloride (TMA), tert-butylphosphonic acid (TBPA).

Aluminum rods embedded in a Teflon sheath (99.999\%) and aluminum plates (5050-H24) were used as substrates and coated using various sol-gel formulations (Table 6) via dip-coating. The substrates were then submerged in $100 \mathrm{ppm} \mathrm{NaCl}$ solution at $25^{\circ} \mathrm{C}$ for $20 \mathrm{~min}$ and subjected to ENM to study the initiation and propagation of corrosion at the interface of aluminum plates and coating. It is considered that the higher the value of $R_{n}$, the better the corrosion resistance of the coating. The results showed that PTMOS + PPA sol-gel coatings exhibited the best protection $\left(R_{n}=6.0 \mathrm{k} \Omega\right)$ compared to uncoated aluminum $\left(R_{n}=1.5 \mathrm{k} \Omega\right)$ and PTMOS coatings $\left(R_{n}=4.0 \mathrm{k} \Omega\right)$.

In potentiodynamic polarization measurements, aluminum in the form of rods was used. These rods were exposed to $100 \mathrm{ppm} \mathrm{NaCl}$ solution for $30 \mathrm{~min}$ before the measurements. For these measurements, as mentioned before, the more positive $E_{\text {corr }}$ and the lower the current values, the better the corrosion resistance. PTMOS + PPA showed the highest $E_{\text {corr }}$ value and a low current. In contrast, the TEOS-based film showed a negative $E_{\text {corr }}$ value, which indicated the poor corrosion resistance. Even addition of TMA in TEOS film accelerated the corrosion process. Potentiodynamic polarization measurements of samples for electrodeposition process showed slight enhancement of corrosion inhibition. Elemental analysis was used to detected phosphorus (due to PPA) in the films. For PTMOS based film, phosphorus was detected and it was hypothesized that the anion of PPA and phenyl group of PTMOS tends to form stable $\pi$-interactions in the sol-gel system [22]. 


\subsubsection{Vinylphosphonic Acid (VPA)}

Cooling of an aluminum alloy plate heat exchanger by seawater needs superior corrosion resistance. Trifluoroethylene polymers were preferred to form a fluorocarbon resin coating on aluminum alloy because of high adhesion to the organic phosphonic acid primer coating and high corrosion resistance. Vinylphosphonic acid (VPA) was used as the organic phosphonic acid primer because of its handle and its superior adhesion effect. The aluminum alloy (3003) was first anodically oxidized and then immersed in VPA aqueous solution $(10 \mathrm{~g} / \mathrm{L})$ at $65^{\circ} \mathrm{C}$ for $10 \mathrm{~s}$ or $120 \mathrm{~s}$, followed by fluorocarbon resin coating and dried at $50{ }^{\circ} \mathrm{C}$ for $24 \mathrm{~h}$. For the corrosion test, one hundred $1 \mathrm{~mm}^{2}$ cross-cuts were formed on the coating, and a tape was attached on it and then peeled by the method specified in JIS K 5600-5-6. For samples with coatings without an organic phosphonic acid primer, all hundred cross-cuts of the coating were peeled off $(0 / 100)$ upon tape peeling. However, for the coating with VPA (10 s or 120 s), all 100 cross-cuts remained unpeeled (100/100), which indicated an excellent anti-corrosion property [49].

\subsubsection{1,12-Dodecyldiphosphonic Acid (DDP)}

Self-assembled monolayers (SAMs) consisting of zirconium phosphate and their derivatives were used as the substitute to chromating on aluminum for ecological reasons [8]. Aluminum alloy (1100) was pretreated with $0.4 \mathrm{vol} \%$ of 3-aminopropyltriethoxysilane (APTES) in toluene by chemical vapor deposition. APTES is believed to prevent the formation of defects and provide protection against the harsh subsequent phosphonation treatment $(\mathrm{pH} \leq 2)$. Subsequently, the substrate was immersed in $0.2 \mathrm{~mol} \mathrm{POCl}_{3}$ and $\gamma$-collidine of acetonitrile solution. After ultrasonically rinsing with acetonitrile, the substrate was dipped in $5 \mathrm{mmol} / \mathrm{dm}^{3}$ zirconyl chloride $\mathrm{ZrCl}_{2} \cdot 8 \mathrm{H}_{2} \mathrm{O}$ of ethyl alcohol-aqueous solution to form a zirconium phosphate ( $\mathrm{ZrP})$ layer. Ultimately the substrate was suspended in $2 \mathrm{mmol} / \mathrm{dm}^{3}$ DDP of acetone-aqueous solution to form the three layered Zr-DDP coating as shown in Scheme 1.

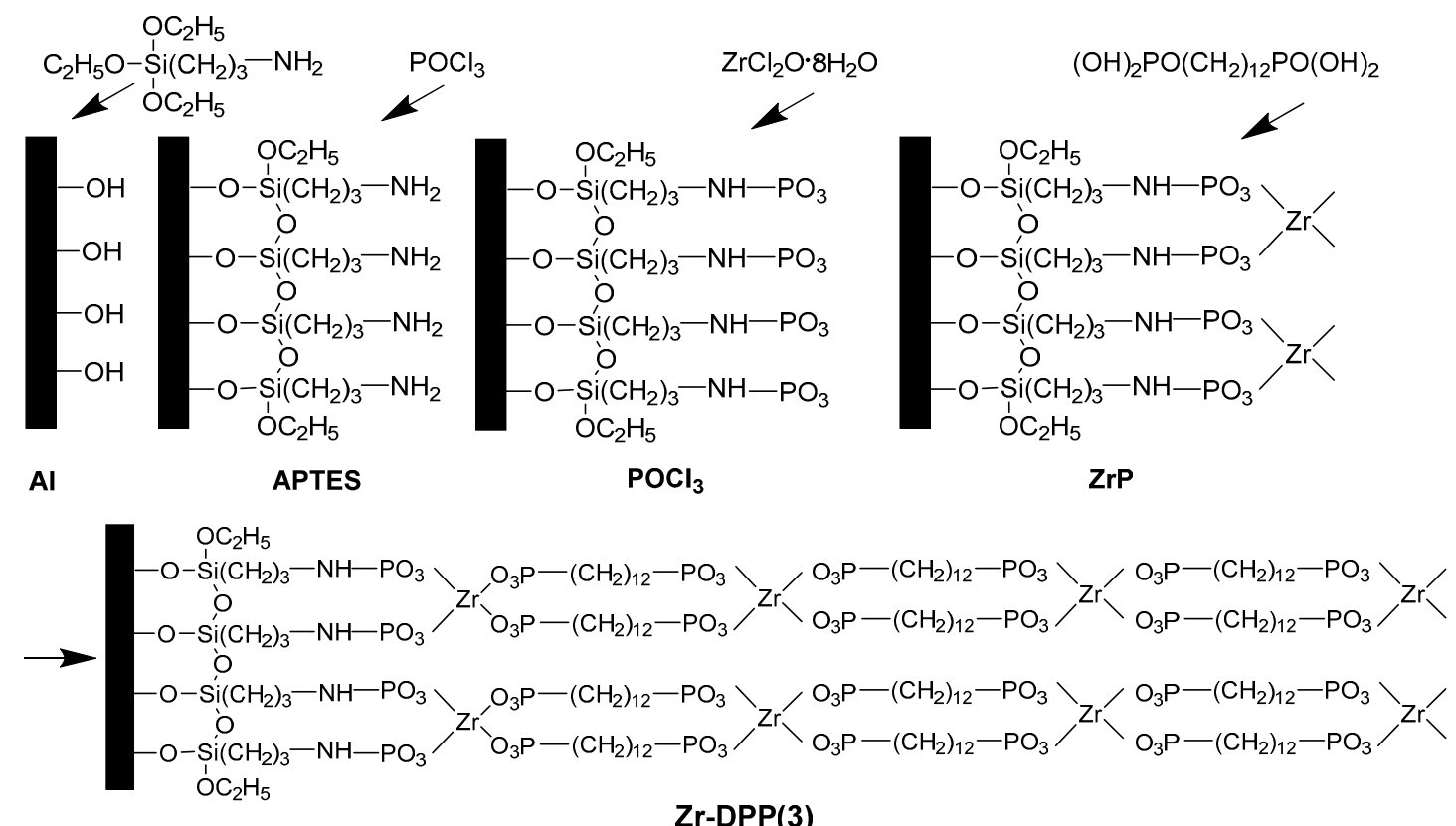

Zr-DPP(3)

Scheme 1. Multilayer zirconium phosphate treatment consisting of DDP. (Reproduced from [8] with permission; Copyright 2003 Elsevier).

The Zr-DDP multi-layers film was tested for corrosion resistance by exposure to $5 \mathrm{wt} \% \mathrm{NaCl}$ solution at $308 \mathrm{~K}$ for $48 \mathrm{~h}$ in the salt-spray test. The corrosion rate of multi-layer Zr-DDP coating is $0 \%$ (indicating no corrosion at all) and showed superior anti-corrosion properties compared to APTES 
coating $(100 \%), \mathrm{POCl}_{3}$ coating $(80 \%)$, and conventional $\mathrm{ZrP}$ coating $(100 \%)$. After a $72 \mathrm{~h}$ salt spray test, $\mathrm{Zr-DDP}$ films were corroded with a corrosion rate of $60 \%$ whereas the other treatments showed $100 \%$ corrosion rate. The authors concluded that Zr-DDP films constructed by SAMs are promising surface finishing treatments to replace the conventional phosphating or chromating treatments [8].

\subsubsection{Amino Trimethylene Phosphonic Acid (ATMP)}

ATMP is known to have anti-scale performance due to its excellent chelating ability, and a low threshold inhibition and lattice distortion process. ATMP was used as an inhibitor to improve the anti-corrosion properties of aluminum alloy (AA2024-T3) [15]. The deposition bath was made by mixing ATMP in distilled water, tetraethylorthosilicate (TEOS), and ethanol in a 6:4:90 $(v / v / v)$ ratio. In this work, the effect of three different pretreatments (acetic acid, acetic acid- $\mathrm{NaOH}, \mathrm{NaOH}$ ) and ATMP concentration on the corrosion protection were studied. SEM and energy-dispersive X-ray spectroscopy (EDS) analysis of the treated aluminum surface confirmed that acetic acid pretreatment of the aluminum surface was the most efficient pretreatment. The authors assumed that acetic acid pretreatment increased surface area of the alloy matrix, which led to more formation of favorable metalosiloxane and metal-phosphonic bonds to the surface.

Three different ATMP concentrations of deposition baths were investigated. The corrosion behavior of the treated aluminum was tested against $0.05 \mathrm{M} \mathrm{NaCl}$ solution for $48 \mathrm{~h}$. EIS was used to assess the corrosion resistance, as mentioned in Section 3.1.1. High $R_{\mathrm{HF}}$ (the resistance of the high frequency related to the coating layer) value and low $\mathrm{CPE}_{\mathrm{HF}}$ (the capacitance of the high frequency related to the coating layer) value indicated higher corrosion resistance. Results of the TEOS coating containing ATMP $\left(5.00 \times 10^{-4} \mathrm{M}\right)$ showed $R_{\mathrm{HF}}\left(\mathrm{kOhm} \cdot \mathrm{cm}^{2}\right): 81.4, \mathrm{CPE}_{\mathrm{HF}}\left(\mu \mathrm{F} \cdot \mathrm{cm}^{-2} \cdot \mathrm{s}^{n-1}\right): 16.2$, whereas increasing or decreasing the ATMP concentration had an adverse effect on corrosion protection. In contrast, TEOS coating without ATMP resulted in $R_{\mathrm{HF}}\left(\mathrm{kOhm} \cdot \mathrm{cm}^{2}\right): 5.06, \mathrm{CPE}_{\mathrm{HF}}\left(\mu \mathrm{F} \cdot \mathrm{cm}^{-2} \cdot \mathrm{s}^{n-1}\right)$ : 160.5 , indicating no corrosion resistance at all. The authors believe that the corrosion protection was caused by the strong chemical bonding of phosphonic groups to the aluminum substrate [15].

\subsubsection{Ethylenediamine Tetra Methylene Phosphonic Acid (EDTPO)}

Modification of aluminum alloy (AA2024) with a well-known anticorrosive epoxy-polyamide paint in combination with vinyltrimethoxysilane (VTMS)/tetraethylorthosilicate (TEOS) nanocoating was used to improve its corrosion resistance and adhesion to organic layers. EDTPO, as a catalyst, was incorporated into VTMS/TEOS to form a sol-gel coating on the substrate surface and is believed to improve the formation of Al-O-P bonds which is beneficial for anticorrosive properties [17]. The concentration of EDTPO in the sol-gel deposition solution was $3.75 \times 10^{-5} \mathrm{~mol} \cdot \mathrm{L}^{-1}$. EIS measurement was carried out in $3.5 \% \mathrm{NaCl}$ aqueous solution. Substrates coated with epoxy and sol-gel film with or without EDTPO showed good corrosion resistance: The EIS results remained constant at $10^{9.5} \Omega \cdot \mathrm{cm}^{-2}$ up to 300 days, which indicated an excellent corrosion resistance. Whereas the values for the epoxy coating without sol-gel film started to decrease after 160 days and 300 days later, coating resistance was only $10^{7.9} \Omega \cdot \mathrm{cm}^{2}$. A cyclic accelerated corrosion test in $3.5 \% \mathrm{NaCl}$ solution for 60 days was evaluated by ASTM D-1654. The results showed that the failure area of the coating with EDTPO was $0.8 \%$, which indicates an excellent corrosion resistance compared to the coating without EDTPO $(1.3 \%)$ or the coating without any sol-gel primer (32\%). After accelerated corrosion, the pull-off test (UNE-EN-ISO 4624) showed that the adhesion reduction for the coating with EDTPO to be $0 \%$. However, the adhesion of coating without EDTPO showed a reduction of $15 \%$, and the adhesion of the coating without any sol-gel primer reduced to $40 \%$. It was concluded that the EDTPO-modified silane coating (VTMS/TEOS) has excellent adhesion and anti-corrosion properties because of its barrier properties [17].

In a similar work, two different concentrations of EDTPO $\left(3.75 \times 10^{-5}\right.$ and $\left.3.75 \times 10^{-4} \mathrm{~mol} \cdot \mathrm{L}^{-1}\right)$ were incorporated in TEOS to form sol-gel deposition solutions, which are named TEOS/EDTPO $10^{-5}$ and TEOS/EDTPO $10^{-4}$, respectively. Aluminum alloy (AA2024-T3) panels were immersed in 
these formulations to form an anti-corrosion film. As mentioned in Section 3.1.1, a high $R_{\mathrm{HF}}$ value and low $\mathrm{CPE}_{\mathrm{HF}}$ value indicates a corrosion resistance. After seven days of soaking in the $0.05 \mathrm{~mol} \cdot \mathrm{L}^{-1}$ $\mathrm{NaCl}$ solution at $25{ }^{\circ} \mathrm{C}$, EIS test results of TEOS/EDTPO $10^{-4}$ was $R_{\mathrm{HF}}\left(\Omega \cdot \mathrm{cm}^{2}\right): 248.3, \mathrm{CPE}_{\mathrm{HF}}$ $\left(\mu \mathrm{F} \cdot \mathrm{cm}^{-2} \cdot \mathrm{s}^{n-1}\right): 1.74$ and for TEOS/EDTPO $10^{-5}$ it was $R_{\mathrm{HF}}\left(\Omega \cdot \mathrm{cm}^{2}\right): 177.4, \mathrm{CPE}_{\mathrm{HF}}\left(\mu \mathrm{F} \cdot \mathrm{cm}^{-2} \cdot \mathrm{s}^{n-1}\right)$ : 15.8. Thus, it was clear that TEOS/EDTPO $10^{-4}$ could provide a better corrosion resistance. The substrate coated with TEOS/EDTPO $10^{-4}$ was further investigated by soaking it in $0.05 \mathrm{~mol} \cdot \mathrm{L}^{-1} \mathrm{NaCl}$ solution at $70{ }^{\circ} \mathrm{C}$ with various immersion times. After a $24 \mathrm{~h}$ exposure, the impedance modulus is lower than the measurements for $25^{\circ} \mathrm{C}$. Nevertheless, the corrosion resistance of the EDTPO containing coatings was still approximately six times higher than for TEOS-only coatings. In this work, it was also confirmed that EDTPO enhanced the corrosion resistance of the sol-gel coating even at a higher temperature [30].

In a separate work done by the same authors, the corrosion behavior of EDTPO and ATMP was compared in TEOS sol-gel coating on the aluminum alloy (AA1100) surface. Substrates were dip-coated in two different solutions: $3.75 \times 10^{-4} \mathrm{~mol} \cdot \mathrm{L}^{-1}$ EDTPO in TEOS ethanol solution and $5.00 \times 10^{-4} \mathrm{~mol} \cdot \mathrm{L}^{-1}$ ATMP in TEOS ethanol solution. EIS was used to evaluate the corrosion of coated substrates in a $0.05 \mathrm{~mol} \cdot \mathrm{L}^{-1} \mathrm{NaCl}$ solution for seven days. The corrosion resistance of TEOS/EDTPO was $R_{\mathrm{HF}}\left(\Omega \cdot \mathrm{cm}^{2}\right): 141.0, \mathrm{CPE} \mathrm{HF}\left(\mu \mathrm{F} \cdot \mathrm{cm}^{-2} \cdot \mathrm{s}^{n-1}\right): 2.45$. For TEOS/ATMP it was $R_{\mathrm{HF}}\left(\Omega \cdot \mathrm{cm}^{2}\right): 113.7$, $\mathrm{CPE}_{\mathrm{HF}}\left(\mu \mathrm{F} \cdot \mathrm{cm}^{-2} \cdot \mathrm{s}^{n-1}\right): 1.65$. It was concluded that both EDTPO and ATMP could provide considerable corrosion protection for AA1100. However, the corrosion resistance of EDTPO-containing coatings was higher than for ATMP-containing coatings [40].

\subsubsection{1,2-Diaminoethanetetrakis-Methylenephosphonic Acid (DETAPO)}

The adhesion and corrosion performance of silane sol-gel film coating on aluminum has been reported. Especially ATMP and EDTPO, as the phosphonic catalysts in a sol-gel system, have been well studied for the protection against corrosion $[15,17,30,40]$. Phosphonic derivative DETAPO, which has a similar structure has also been tested because of higher concentration of $\mathrm{O}=\mathrm{P}-\mathrm{OH}$ group in its structure compared to ATMP and EDTPO [23]. In this work, aluminum alloy (AA2024-T3) was coated with VTMS/TEOS sol-gel film containing DETAPO and the epoxy-polyamide resin. A cyclic accelerated corrosion test in $3.5 \% \mathrm{NaCl}$ for 45 days was performed according to the ASTM D1654 method. The results showed that the failure area of DETAPO modified coating was around $10-11 \%$, compared to the $35 \%$ failure area for the epoxy coating without any silane film. Pull-off tests after accelerated corrosion showed that adhesion reduction of DETAPO-modified silane coating was around $62.5-52.9 \%$, on the other hand, the coating without any silane film reduced to $34.7 \%$ adhesion. It was clear that the DETAPO-modified coating can provide an outstanding corrosion resistance for aluminum surface [23].

\subsection{7. (12-Ethylamino-Dodecyl)-Phosphonic Acid}

Self-assembled monolayers (SAMs) were used to form thin organic adhesion promoter layers to replace the chromating process on aluminum. (12-ethylamino-dodecyl)-phosphonic acid was chosen as a model functional organic phosphonic acid in this work because of its phosphonic acid anchor group which acts as a reactive group for the aluminum surface, its aliphatic part acting as a hydrophobic spacer, and its amino head group serving as a reactive group to organic material [27]. The aluminum alloy (AlMg) was dip-coated in the $10^{-3} \mathrm{M}$ (12-ethylamino-dodecyl)-phosphonic acid solution of ethanol/water (3:1 vol \%). The surface analysis by FTIR and surface plasmon resonance spectroscopy (SPR) confirmed that organophosphonic acid could spontaneously adsorb on the aluminum surface and subsequently form oriented layers. The adhesion promotion and corrosion resistance of the aluminum surface were confirmed by the acetic acid salt spray-test (ASS-test, DIN 50021) and filiform test (DIN 50024). Coated panels were scratched and exposed in a climate chamber of $\mathrm{NaCl}$ solution and acetic acid. After $1200 \mathrm{~h}$ exposure, the infiltrations for the amino phosphonic acid coating was 
less than $1 \mathrm{~mm}$, which indicates excellent corrosion resistance compared to $8 \mathrm{~mm}$ infiltration for the uncoated substrate [27].

\subsubsection{Aminopropyl Phosphonate (APP)}

To replace harmful chromate conversion layers, environmentally-friendly aminopropyl phosphonate (APP), aminopropyl silane (APS), and hexamethyldisiloxane (HMDSO) were used to coat aluminum alloy (6016) [9]. As known, filiform corrosion (FFC) usually occurs at the interface of a polymer and an aluminum alloy. Hence, the corrosion resistance and adhesion promotion of these coatings were studied to find a suitable pretreatment for automotive applications. The influence of the rolling direction on filiform corrosion was also investigated. Substrates were dipped in $1 \mathrm{mmol}$ APP solution with $\mathrm{pH} 7$ for $1 \mathrm{~h}$ followed by an epoxy coating. APS was coated on substrates with the same dip-coating method and, for HMDSO, a plasma coating technique was used. Before the corrosion test, substrates were scribed perpendicularly or parallel to the rolling direction. The corrosion test was carried out by soaking scratched substrates in a vessel with concentrated $\mathrm{HCl}$ vapor for $20 \mathrm{~min}$. FFC was evaluated based on filament initiating time and the number of filaments observed in the digital microscope. From visual observation, it could be confirmed that filaments grew predominantly along the rolling direction, which indicated that the rolling direction had an influence on the interfacial bonding. The FFC severities were as follows: APS > etching $>$ APP $>$ HMDSO plasma. The adhesion of the epoxy adhesive to the substrate was analyzed by a peeling test, and the results follow a trend for different coatings: HMDSO plasma > APS > APP > etching. It was concluded that the adhesion of the coating had a great influence on filament morphology and further on filament propagation, but an increase in adhesion of the coating was not a guarantee of resistance to FFC [9].

The corrosion performance of aluminum powder was enhanced by a novel functionalization with graphene oxide (GO) with phosphonic acid as a linker [11]. APP was used as the "link" agent to connect graphene oxide (GO) with aluminum powder. APP was added in a suspension of GO and deionized water. After stirring, refluxing, dialyzing, and drying, GO-APP was added in the aluminum powder suspension to form the oxide modified aluminum powder (GO-Al). Corrosion performance was tested in dilute hydrochloric acid. Since corrosion of aluminum generated hydrogen, corrosion behavior could be evaluated by detecting the amount and the yield time of hydrogen gas. The results showed that for the $\mathrm{Al} / \mathrm{HCl}$ system, the hydrogen was detected after $100 \mathrm{~min}$. However, in the GO-Al/ $\mathrm{HCl}$ system, hydrogen was detected only after $140 \mathrm{~min}$, which confirms enhanced the anti-corrosive performance of GO-Al. Through XPS, FTIR, field emission scanning electron microscopy (FE-SEM), and EDS, it could be confirmed that the flaky aluminum particle was successfully covalently bonded and covered by GO. In conclusion, GO as a barrier coating was well connected with epoxy through APP [11].

\subsection{9. $\omega$-(3-Phenylpyrrol-1-ylalkyl) Phosphonic Acid (Cn-Ph-P)}

$\mathrm{Cn}$-Ph-P was used to build SAMs on the aluminum surface $\left(\mathrm{Al} / \mathrm{Al}_{2} \mathrm{O}_{3}\right.$ chemical vapor deposition on polished, p-doped Si wafers) because its phosphonic acid group could anchor to the aluminum surface and its pyrrole group could be used to achieve an in situ surface polymerization with further monomers [10]. The substrates were immersed in a $\mathrm{C} n$-Ph-P solution for different time intervals ( $5 \mathrm{~min}$, $1 \mathrm{~h}$, and $24 \mathrm{~h}$ ) to form SAMs on the surface. After that, the pyrrole monomer was polymerized with the free terminal pyrrole group of the SAM by oxidants, like sodium or ammonium peroxidisulphate, followed by the growth of polypyrrole (PPY) on the surface. The contact angle was used to evaluate the adsorption behavior. It was inferred that a more hydrophobic surface (higher contact angles) was caused by phosphonic acid group reacting with surface and the terminal polymerizable group present on top of it. The surface contact angle rose up after adsorption of $\mathrm{C} n \mathrm{PhP}$, which indicated that $\mathrm{C} n \mathrm{PhP}$ was oriented on the surface. The contact angles of $\mathrm{C} 12 \mathrm{PhP}$ were slightly higher than $\mathrm{C} 10 \mathrm{PhP}$ because of the stronger van der Waals interaction between the alkyl chains. $\mathrm{C} 12 \mathrm{PhP}$ showed excellent stability after eight repeated cycling using the Wilhelmy method [10]. 


\subsubsection{0. $\omega$-(2,5-Dithienylpyrrol-1-yl-alkyl) Phosphonic Acid (SNS- $n$-P)}

SNS- $n$-P as a model functional phosphonic acid was used to study the influence of varying alkyl chain length (SNS10P, SNS4P) to the adsorption on the aluminum surface ( $\mathrm{Al} / \mathrm{Al}_{2} \mathrm{O}_{3}$ chemical vapor deposition on polished, p-doped Si wafers). The contact angle of SNS10P coated surface did not change after ten repeated cycles by the Wilhelmy method, which indicated that SNS10P had a very stable bond to the surface. However, the contact angle of SNS4P coating decreased significantly with the increasing number of repeated cycles. Thus, this comparison of analogue structures SNS10P and SNS4P showed that the alkyl chain length of the structures influences the bonding at the surface [10].

\subsubsection{1. (1H,1H, 2H,2H-Heptadecafluorodec-1-yl) Phosphonic Acid (HDF-PA)}

Trichlorosilane or metal-ligand coordination has mostly been used to engineer surface wetting properties on metals. However, they were highly moisture sensitive due to hydrolysis and self-condensation of silanes [51-53]. Therefore, HDF-PA was employed to coat different self-assembled monolayers (SAMs) on metal oxide surfaces because phosphonic acid-functionalized molecules were stable in water, and built a stable metal-ligand coordination and hetero-condensation between phosphonate and substrate surfaces. Substrates with a $30 \mathrm{~nm}$ thick $\mathrm{Al}_{2} \mathrm{O}_{3}$ film were dip-coated in $2 \mathrm{mM}$ HDF-PA isopropyl alcohol for $1 \mathrm{~h}$. After the water flow test, changes in surface contact angle were analyzed to evaluate the reliability of the chemical bond between SAMs and substrate surface. (Heptadecafluoro-1,1,2,2-tetrahydrodecyl) trichlorosilane (HDF-S) was also studied as a comparison. The bare $\mathrm{Al}_{2} \mathrm{O}_{3}$ films showed contact angle values of $70.0^{\circ} \pm 1.5^{\circ}$, the contact angles of HDF-PA and HDF-S-coated $\mathrm{Al}_{2} \mathrm{O}_{3}$ films were enhanced to $99.9^{\circ} \pm 1.0^{\circ}$ and $102.7^{\circ} \pm 2.4^{\circ}$. After exposing the surface to $5 \mathrm{~L}$ of water droplets, contact angles of HDF-S coating remained around $102.7^{\circ}$, whereas the contact angles of HDF-PA coating reduced from $99.9^{\circ}$ to $69.3^{\circ}$. The results showed that HDF-S formed a more stable bond than HDF-PA, which may be due to the cross-linked siloxane network on the surface. A low-temperature $\left(<150^{\circ} \mathrm{C}\right)$ thermal annealing process was used to improve the HDF-PA bond formation. The results showed that after the water flow test, contact angles for $100{ }^{\circ} \mathrm{C}$ and $150{ }^{\circ} \mathrm{C}$ annealed substrates were stable at around $101^{\circ} \mathrm{C}$. Therefore, the durability of HDF-PA coatings could be improved by an additional thermal annealing process at $100-150{ }^{\circ} \mathrm{C}[19]$.

\subsubsection{Poly (Vinyl Phosphonic Acid) (PvPA)}

PvPA was coated as a thin polymeric interfacial layer on aluminum alloy (AA 1050) surface by dip-coating and compared to the poly (acrylic acid) (PAA), poly (ethylene-alt-maleic anhydride) (PEMah) coated aluminum surface. After that, an epoxy (Resolution Epikote 1001) adhesive was coated on the modified substrate. The PvPA-based system showed poor adhesion performance in the pull-off test compared to PEMah, because PvPA forms a weakly-cured epoxy/polymer interphase [24].

\subsection{Phosphates Used as Pre-Coating}

\subsubsection{Monostearyl Acid Phosphate (MSAP)}

A novel ultrasonic assisted deposition (USAD) method was used to coat phosphate films on aluminum and compared with films formed by mild mechanical stirring [12]. MSAP was used in this study and its impact on the adsorption behavior of these films was evaluated. Simultaneously, the corrosion resistance and adhesion properties of the coatings were studied as well. Infrared spectroscopy (IR) and NMR were used to analyze the stability of the MSAP film, after soaking the substrates for $5 \mathrm{~h}$ at $100{ }^{\circ} \mathrm{C}$ in a reflux distillation column containing water and steam. It was observed in NMR spectra that the USAD substrates had resolved peaks at -7 and -14 ppm, whereas the chemical shift of the stirring coated substrates occurred at $0 \mathrm{ppm}$. Based on the previous NMR studies for alkylphosphate films on metal oxide surfaces [54-58], it was confirmed that the peaks at -7 and -14 ppm meant bidentate and tridentate interactions between the aluminum oxide surface and the phosphate. It was 
concluded that the USAD treatment favored tridentate interaction between MSAP and aluminum oxide [12].

\subsubsection{Phosphoric Acid Mono-(12-Hydroxy-Dodecyl) Ester}

From earlier studies, it was clear that cooperated pretreatments of phosphoric acid anodic oxidation (PAA) and an adhesion promoter (AP) (phosphoric acid mono alkyl ester) could enhance the adhesion and durability of the aluminum/epoxy bonding and thus could be considered as an alternative to the chromium-based pretreatment for aluminum [59]. In a more recent work, aluminum substrates were first anodized in a phosphoric acid solution to create PAA. Then the PPA substrates were dip-coated in $10^{-3} \mathrm{M}$ aqueous solution of phosphoric acid mono-(12-hydroxy-dodecyl) ester for $5 \mathrm{~min}$ at room temperature [41]. EIS and the floating roller peel test were used to study the corrosion performance of PAA and PAA/AP systems. After $48 \mathrm{~h}$ soaking in $3 \% \mathrm{NaCl}$ solution at $80{ }^{\circ} \mathrm{C}$, the EIS results showed that the PAA and PAA/AP pretreated surfaces had a strong influence on the crosslinking of the epoxy at the interphase. For the floating roller peel test, one rigid and one flexible coated substrate was first bonded with Delo-Duopox 1891 adhesive. The joints were then corroded according to accelerated method VDA 621-415. The peel test was performed based on DIN EN 1464. The results indicated that after aging, PAA/AP treated substrates showed higher peel strength (around $3.3 \mathrm{~N} / \mathrm{mm}$ ) than PAA treated substrates (around $2.8 \mathrm{~N} / \mathrm{mm}$ ). It was confirmed that AP could enhance the cross-linking of the epoxy coat. The authors suggested that the AP layer was well-ordered on the surface and the strong bonding of the phosphoric acid anchor group to PAA led to the good adhesion and corrosion resistance [41].

\subsubsection{Fluoro Alkyl Phosphates (Zonyl UR)}

Zonyl UR was deposited on aluminum plate and aluminum oxide powder as a corrosion inhibitor and adhesion promoter. Two different deposition methods were compared: The ultrasonic assisted deposition (USAD) or magnetic stirring. Environmental stability of the coating was observed in a reflux distillation column $\left(100^{\circ} \mathrm{C}\right)$. For aluminum plate, the change in IR spectra was used to evaluate the stability of the coatings, which showed that after $5 \mathrm{~h}$ refluxing, barely any changes occurred to the USAD-coated surface. In contrast, clear evidence of corrosion was observed for stirring deposition. For aluminum powder, IR spectra of stirring deposition exhibited broad features, which did not exist in USAD. Solid state nuclear magnetic resonance (NMR) was used to study the interactions between the phosphate group and the aluminum oxide powder. The results confirmed that USAD could provide a more homogeneous film with mostly tridentate interaction and low coverage of mono- and bidentate interaction [12].

\subsection{Coatings Dissolved in Paints that Contain Phosphonic Acids or Phosphates}

\subsubsection{Phenylphosphonic Acid (PPA)}

PPA has been used as a self-phosphating agent and an acid catalyst in the formulation of a polyester-melamine coating for aluminum surface [14]. In this work, a chrome-free single-step in situ phosphatizing coating (ISPC) was used on aluminum alloy (3003 or 3105). This work is based on a patent published earlier, where for an ISPC, an optimum amount of an in situ phosphatizing reagent (ISPR) or a mixture of ISPRs is predisposed in the paint system to form a stable and compatible coating formulation [60]. It is believed that when a chrome-free single-step coating of the in situ self-phosphating paint is applied to a bare metal substrate, the phosphatizing reagent chemically and/or physically reacts in situ with the metal surface to produce a metal phosphate layer and simultaneously forms covalent $\mathrm{P}-\mathrm{O}-\mathrm{C}$ (phosphorus-oxygen-carbon) linkages with the polymer resin. Such linkages enhance the adhesion of the coating and suppress substrate corrosion. It was assumed that PPA could also provide phosphate formation on the metal surface in the in-situ phosphatizing treatment [14]. The aluminum panels were spray coated with polyester-melamine paint containing 
$1 \mathrm{wt} \%$ PPA and other formulations containing commercially available acid sources (phosphonosuccinic acid, para toluene sulphonic acid). Coated substrates were analyzed for corrosion behavior using EIS measurements, saltwater immersion, and pencil hardness tests. The paint on cured panels was removed by a razor and analyzed for the glass transition temperature $\left(T_{g}\right)$ using differential scanning calorimetry (DSC). A higher $T_{g}$ correlates with a coating with a higher cross-linking density in the polymer. $T_{g}$ of $1 \mathrm{wt} \%$ PPA paint formulation was $22{ }^{\circ} \mathrm{C}$. After the paint was exposed to $300{ }^{\circ} \mathrm{C}, T_{g}$ increased to $65^{\circ} \mathrm{C}$ indicating an increase in the crosslinking density of the polymer. For formulations containing para toluene sulphonic acid (commercial solution), the crosslinking density dropped as the $T_{g}$ dropped after severe heating. This resulted from the possible cleavage of polyester-melamine cross-links in favor of the melamine self-condensation in the paint films [61].

After immersion of the coated substrate in $3 \% \mathrm{NaCl}$ for three days, EIS was used to study the corrosion behavior. It is known from previous work that a surface with an impedance at the low frequency below $10^{7} \Omega \cdot \mathrm{cm}^{2}$ is considered a poor protective barrier [44]. The paint with $1 \mathrm{wt} \%$ PPA resulted in the impedance value of $10^{10} \Omega \cdot \mathrm{cm}^{2}$ at low frequency. This result indicates the superior corrosion protection of PPA-sol-gel coatings. For saltwater immersion test, test panels were scribed with an " $\mathrm{X}$ " and then were soaked in a $3 \% \mathrm{NaCl}$ solution for 66 days. After that, a tape was firmly pressed against the scribed area and pulled to remove. The saltwater corrosion test was evaluated using the ASTM D3359A method, which showed that there was no discoloration of the paint and no paint was removed with the tape. Only very few tiny blisters $(\varnothing<1 \mathrm{~mm})$ around the scribe were observed, which may result from paint defects. The pencil hardness of the paint (ASTM D3363) was F. The paint was without any discoloration. The authors concluded the paint with $1 \mathrm{wt} \%$ of PPA provided excellent corrosion resistance [14].

\subsubsection{2-(Phosphonooxy) Benzoic Acid (Fosfosal)}

Fosfosal was used as an acid additive in the polyester-melamine paint and was used in the in situ phosphatizing treatment on aluminum alloy (3003) [14]. Substrates were spray-coated with polyester-melamine paint containing different concentrations $(0.5 \%, 0.75 \%$, and $1 \%)$ of Fosfosal. DSC was used to evaluate the cross-linking density of polymer by analyzing the glass transition temperature $\left(T_{g}\right)$. The DSC program involved annealing at $80^{\circ} \mathrm{C}$, then scanning from $-50{ }^{\circ} \mathrm{C}$ to $300{ }^{\circ} \mathrm{C}$ with a heating rate of $10^{\circ} \mathrm{C} / \mathrm{min}$. Higher $T_{g}$ means higher cross-linking density. EIS was used to study the corrosion performance of coated substrates after three days in $3 \% \mathrm{NaCl}$ immersion. As mentioned earlier, EIS results of lower than $10^{7} \Omega \cdot \mathrm{cm}^{2}$ at low frequency meant poor anti-corrosion properties. Corrosion resistance was further studied after saltwater immersion. Coated substrates were scribed with an " $\mathrm{X}$ " and immersed in $3 \% \mathrm{NaCl}$ solution for 66 days. After drying, a tape was firmly pressed against the " $X$ " area and pulled to remove. The corrosion resistance was evaluated by ASTM method D3359 A The pencil hardness of the painted Al panels was measured using ASTM method D3363. The results of the corrosion test are summarized in Table 7.

Table 7. Measurement results of different Fosfosal concentrations.

\begin{tabular}{ccccc}
\hline Concentration & DSC $\left(\boldsymbol{T}_{\boldsymbol{g}}\right)$ & EIS $\left(\boldsymbol{\Omega} \cdot \mathrm{cm}^{2}\right)$ & Saltwater Immersion & Pencil Hardness \\
\hline $1 \%$ & 35 to $59^{\circ} \mathrm{C}$ & $10^{9}-10^{7}$ & - & $4 \mathrm{H}$ \\
$0.75 \%$ & 34 to $55^{\circ} \mathrm{C}$ & $10^{9}$ & $\varnothing 3 \mathrm{~mm}$ blister & $4 \mathrm{H}$ \\
$0.5 \%$ & 18 to $48^{\circ} \mathrm{C}$ & $10^{9}$ & - & $\mathrm{HB}$ \\
\hline
\end{tabular}

The corrosion resistance (saltwater immersion) for 1\% Fosfosal coatings could not be estimated with the ASTM method. For 0.5\% Fosfosal, saltwater immersion results were not reproducible; one panel had no paint removal and was evaluated as 5A in the ASTM D3359 A test. One panel had a clump of blisters of size $\sim \varnothing 2 \mathrm{~mm}$ and the rest had no blistering. DSC results for coatings with $0.5 \%$ Fosfosal showed that cross-linking density increased and EIS results showed $0.5 \%$ Fosfosal provided sufficient corrosion protection. In case of pencil hardness test, coatings containing $1 \%$ and $0.75 \%$ 
Fosfosal $(4 \mathrm{H})$ were harder than for $0.5 \%$ Fosfosal (HB). However, EIS results for coatings containing $1 \%$ Fosfosal were not reproducible and saltwater immersion results of $0.75 \%$ Fosfosal were worse than for $0.5 \%$ Fosfosal. Therefore, the authors suggested that $0.5 \%$ Fosfosal should be used in the in situ phosphatizing treatment [14].

\subsubsection{Phosphonosuccinic Acid (PPSA)}

PPSA, as an acid additive, was added to the polyester-melamine paint coated on the aluminum alloy (3003) surface. The EIS accelerated corrosion test was used to study the corrosion performance of these coatings. Polyester-melamine paint with 5\% PPSA could not be fully cured. Thus, paint with $1 \mathrm{wt} \%$ PPSA was coated on the substrate and cured, but these coatings exhibited poor coating properties. EIS results showed that the impedance at low frequency was in the order of $10^{6} \Omega \cdot \mathrm{cm}^{2}$. After one week immersion in $3 \%$ salt solution, many blisters were observed on the paint surface. PPSA performed badly as a catalyst in the polyester-melamine paint system [14].

\section{Conclusions}

Environmental issues with existing pretreatments for aluminum, together with the need to develop new materials with higher mechanical and chemical performance, is driving the research and industrial communities to develop new pretreatments based on phosphonic acids. The new treatments are shown to have higher mechanical performance and corrosion protection; however, much work in adaptation to industrial processes and their real environmental impact need to be evaluated to demonstrate the commercial feasibility of these treatments. In many cases, the phosphonic acids are incorporated in sol-gel coatings, which look simple in batch-scale application in laboratory research. However, it is not clear if such treatments can be adapted to continuous handling in industrial production. The use of hybrid systems, such as phosphonic acid-modified graphene oxide, is quite promising. However, the economics of such treatments will determine its potential commercial exploitation. We believe that the combination of various organic and inorganic conversion technologies for aluminum is needed to obtain industrially-feasible solutions. The development of novel phosphonic acid chemistry with a focus on self-healing, superior galvanic protection, and higher mechanical performance will have a strong focus in the future. New phosphonic acids should be screened for their toxicological profile and environmental impact before any possible commercial exploitation.

Acknowledgments: We would like to acknowledge the financial support of KTI, Switzerland (TISBA project: 16589.1 PFIW-IW) and Novelis Switzerland.

Author Contributions: Ruohan Zhao as first Author conducted the literature search. All three authors were involved in the writing and editing of the Manuscript .

Conflicts of Interest: The authors declare no conflict of interest.

\section{References}

1. Twite, R.L.; Bierwagen, G.P. Review of alternatives to chromate for corrosion protection of aluminum aerospace alloys. Prog. Org. Coat. 1998, 33, 91-100. [CrossRef]

2. Liu, Y.; Sun, D.; You, H.; Chung, J.S. Corrosion resistance properties of organic-inorganic hybrid coatings on 2024 aluminum alloy. Appl. Surf. Sci. 2005, 246, 82-89. [CrossRef]

3. Bulusu, A.; Paniagua, S.A.; MacLeod, B.A.; Sigdel, A.K.; Berry, J.J.; Olson, D.C.; Marder, S.R.; Graham, S. Efficient modification of metal oxide surfaces with phosphonic acids by spray coating. Langmuir 2013, 29, 3935-3942. [CrossRef] [PubMed]

4. Queffélec, C.; Petit, M.; Janvier, P.; Knight, D.A.; Bujoli, B. Surface modification using phosphonic acids and esters. Chem. Rev. 2012, 112, 3777-3807. [CrossRef] [PubMed]

5. Pellerite, M.J.; Dunbar, T.D.; Boardman, L.D.; Wood, E.J. Effects of fluorination on self-assembled monolayer formation from alkanephosphonic acids on aluminum: Kinetics and structure. J. Phys. Chem. B 2003, 107, 11726-11736. [CrossRef] 
6. Attavar, S.; Diwekar, M.; Linford, M.R.; Davis, M.A.; Blair, S. Passivation of aluminum with alkyl phosphonic acids for biochip applications. Appl. Surf. Sci. 2010, 256, 7146-7150. [CrossRef]

7. Hoque, E.; DeRose, J.A.; Kulik, G.; Hoffmann, P.; Mathieu, H.J.; Bhushan, B. Alkylphosphonate modified aluminum oxide surfaces. J. Phys. Chem. B 2006, 110, 10855-10861. [CrossRef] [PubMed]

8. Shida, A.; Sugimura, H.; Futsuhara, M.; Takai, O. Zirconium-phosphate films self-assembled on aluminum substrate toward corrosion protection. Surf. Coat. Technol. 2003, 169-170, 686-690. [CrossRef]

9. Liu, X.F. Filiform corrosion attack on pretreated aluminum alloy with tailored surface of epoxy coating. Corros. Sci. 2007, 49, 3494-3513. [CrossRef]

10. Jaehne, E.; Oberoi, S.; Adler, H.-J.P. Ultra thin layers as new concepts for corrosion inhibition and adhesion promotion. Prog. Org. Coat. 2008, 61, 211-223. [CrossRef]

11. He, L.; Zhao, Y.; Xing, L.; Liu, P.; Wang, Z.; Zhang, Y.; Liu, X. Preparation of phosphonic acid functionalized graphene oxide-modified aluminum powder with enhanced anticorrosive properties. Appl. Surf. Sci. 2017, 411, 235-239. [CrossRef]

12. Shepard, M.J.; Comer, J.R.; Young, T.L.; McNatt, J.S.; Espe, M.P.; Ramsier, R.D.; Robinson, T.R.; Nelson, L.Y. Organophosphate Adsorption on Metal Oxide Surfaces; VSP BV: Oud-Beijerland, The Netherlands, 2004; pp. 225-239.

13. Ulman, A. Formation and structure of self-assembled monolayers. Chem. Rev. 1996, 96, 1533-1554. [CrossRef] [PubMed]

14. Whitten, M.C.; Burke, V.J.; Neuder, H.A.; Lin, C.-T. Simultaneous acid catalysis and in situ phosphatization using a polyester-melamine paint: A surface phosphatization study. Ind. Eng. Chem. Res. 2003, 42, 3671-3679. [CrossRef]

15. Dalmoro, V.; Santos, J.H.Z.; Azambuja, D.S. Corrosion behavior of AA2024-T3 alloy treated with phosphonate-containing TEOS. J. Solid State Electrochem. 2012, 16, 403-414. [CrossRef]

16. Jalili, M.; Rostami, M.; Ramezanzadeh, B. Surface modification of aluminum flakes with amino trimethylene phosphonic acid: Studying the surface characteristics and corrosion behavior of the pigment in the epoxy coating. Corrosion 2015, 71, 628-640. [CrossRef]

17. Dalmoro, V.; Aleman, C.; Ferreira, C.A.; dos Santos, J.H.Z.; Azambuja, D.S.; Armelin, E. The influence of organophosphonic acid and conducting polymer on the adhesion and protection of epoxy coating on aluminium alloy. Prog. Org. Coat. 2015, 88, 181-190. [CrossRef]

18. Blajiev, O.L.; Ithurbide, A.; Hubin, A.; Van Haesendonck, C.; Terryn, H. XPS study of the assembling morphology of 3-hydroxy-3-phosphono-butyric acid tert-butyl ester on variously pretreated Al surfaces. Prog. Org. Coat. 2008, 63, 272-281. [CrossRef]

19. Lee, J.; Bong, J.; Ha, Y.-G.; Park, S.; Ju, S. Durability of self-assembled monolayers on aluminum oxide surface for determining surface wettability. Appl. Surf. Sci. 2015, 330, 445-448. [CrossRef]

20. Davies, P.R.; Newton, N.G. The chemisorption of organophosphorus compounds at an Al (111) surface. Appl. Surf. Sci. 2001, 181, 296-306. [CrossRef]

21. Hauffman, T.; Hubin, A.; Terryn, H. Study of the self-assembling of n-octylphosphonic acid layers on aluminum oxide from ethanolic solutions. Surf. Interface Anal. 2013, 45, 1435-1440. [CrossRef]

22. Sheffer, M.; Groysman, A.; Starosvetsky, D.; Savchenko, N.; Mandler, D. Anion embedded sol-gel films on al for corrosion protection. Corros. Sci. 2004, 46, 2975-2985. [CrossRef]

23. Iribarren-Mateos, J.I.; Buj-Corral, I.; Vivancos-Calvet, J.; Aleman, C.; Iribarren, J.I.; Armelin, E. Silane and epoxy coatings: A bilayer system to protect aa2024 alloy. Prog. Org. Coat. 2015, 81, 47-57. [CrossRef]

24. Van den Brand, J.; Van Gils, S.; Beentjes, P.C.J.; Terryn, H.; Sivel, V.; de Wit, J.H.W. Improving the adhesion between epoxy coatings and aluminum substrates. Prog. Org. Coat. 2004, 51, 339-350. [CrossRef]

25. Essahli, M.; El Asri, M.; Boulahna, A.; Zenkouar, M.; Viguier, M.; Hervaud, Y.; Boutevin, B. New alkylated and perfluoroalkylated phosphonic acids: Synthesis, adhesive and water-repellent properties on aluminum substrates. J. Fluorine Chem. 2006, 127, 854-860. [CrossRef]

26. Kowalik, T.; Adler, H.J.P.; Plagge, A.; Stratmann, M. Ultrathin layers of phosphorylated cellulose derivatives on aluminium surfaces. Macromol. Chem. Phys. 2000, 201, 2064-2069. [CrossRef]

27. Adler, H.-J.P.; Jaehne, E.; Stratmann, M.; Grundmeier, G. New concepts for corrosion inhibition and adhesion promotion. Proc. Annu. Meet. Tech. Program FSCT 2002, 80, 528-545.

28. Hofer, R.; Textor, M.; Spencer, N.D. Alkyl phosphate monolayers, self-assembled from aqueous solution onto metal oxide surfaces. Langmuir 2001, 17, 4014-4020. [CrossRef] 
29. Maxisch, M.; Thissen, P.; Giza, M.; Grundmeier, G. Interface chemistry and molecular interactions of phosphonic acid self-assembled monolayers on oxyhydroxide-covered aluminum in humid environments. Langmuir 2011, 27, 6042-6048. [CrossRef] [PubMed]

30. Dalmoro, V.; dos Santos, J.H.Z.; Armelin, E.; Alemán, C.; Azambuja, D.S. Phosphonic acid/silica-based films: A potential treatment for corrosion protection. Corros. Sci. 2012, 60, 173-180. [CrossRef]

31. Guerrero, G.; Chaplais, G.; Mutin, P.H.; Le Bideau, J.; Leclercq, D.; Vioux, A. Grafting of alumina by diphenylphosphinate coupling agents. Mater. Res. Soc. Symp. Proc. 2001, 628. [CrossRef]

32. Guerrero, G.; Mutin, P.H.; Vioux, A. Organically modified aluminas by grafting and sol-gel processes involving phosphonate derivatives. J. Mater. Chem. 2001, 11, 3161-3165. [CrossRef]

33. Mutin, P.H.; Guerrero, G.; Vioux, A. Organic-inorganic hybrid materials based on organophosphorus coupling molecules: From metal phosphonates to surface modification of oxides. C. R. Chim. 2003, 6, 1153-1164. [CrossRef]

34. Textor, M.; Ruiz, L.; Hofer, R.; Rossi, A.; Feldman, K.; Hahner, G.; Spencer, N.D. Structural chemistry of self-assembled monolayers of octadecylphosphoric acid on tantalum oxide surfaces. Langmuir 2000, 16, 3257-3271. [CrossRef]

35. Alexander, M.R.; Thompson, G.E.; Beamson, G. Characterization of the oxide/hydroxide surface of aluminium using x-ray photoelectron spectroscopy: A procedure for curve fitting the $\mathrm{O}$ 1s core level. Surf. Interface Anal. 2000, 29, 468-477. [CrossRef]

36. Jeurgens, L.P.H.; Sloof, W.G.; Tichelaar, F.D.; Mittemeijer, E.J. Composition and chemical state of the ions of aluminium-oxide films formed by thermal oxidation of aluminium. Surf. Sci. 2002, 506, 313-332. [CrossRef]

37. Paniagua, S.A.; Hotchkiss, P.J.; Jones, S.C.; Marder, S.R.; Mudalige, A.; Marrikar, F.S.; Pemberton, J.E.; Armstrong, N.R. Phosphonic acid modification of indium-tin oxide electrodes: Combined XPS/UPS/contact angle studies. J. Phys. Chem. C 2008, 112, 7809-7817. [CrossRef]

38. Van den Brand, J.; Sloof, W.G.; Terryn, H.; de Wit, J.H.W. Correlation between hydroxyl fraction and O/Al atomic ratio as determined from XPS spectra of aluminium oxide layers. Surf. Interface Anal. 2004, 36, 81-88. [CrossRef]

39. Gouzman, I.; Dubey, M.; Carolus, M.D.; Schwartz, J.; Bernasek, S.L. Monolayer vs. multilayer self-assembled alkylphosphonate films: X-ray photoelectron spectroscopy studies. Surf. Sci. 2006, 600, 773-781. [CrossRef]

40. Dalmoro, V.; dos Santos, J.H.Z.; Armelin, E.; Alemán, C.; Azambuja, D.S. A synergistic combination of tetraethylorthosilicate and multiphosphonic acid offers excellent corrosion protection to AA1100 aluminum alloy. Appl. Surf. Sci. 2013, 273, 758-768. [CrossRef]

41. Phung, L.H.; Kleinert, H.; Fuessel, U.; Duc, L.M.; Rammelt, U.; Plieth, W. Influence of self-assembling adhesion promoter on the properties of the epoxy/aluminum interphase. Int. J. Adhes. Adhes. 2004, 25, 239-245. [CrossRef]

42. Rossi, S.; Fedel, M.; Deflorian, F.; Vadillo, M.D. Localized electrochemical techniques: Theory and practical examples in corrosion studies. C. R. Chim. 2008, 11, 984-994. [CrossRef]

43. Jabeera, B.; Shibli, S.M.A.; Anirudhan, T.S. Synergistic inhibitive effect of tartarate and tungstate in preventing steel corrosion in aqueous media. Appl. Surf. Sci. 2006, 252, 3520-3524. [CrossRef]

44. Leidheiser, H., Jr. Towards a better understanding of corrosion beneath organic coatings. Corrosion 1983, 39, 189-201. [CrossRef]

45. Zhou, L.X.; Chen, J.; Yang, Z.R. Methods for study and evaluation of anticorrosion performance of organic coatings. Corros. Sci. Prot. Tech. 2004, 16, 375-377.

46. Bertocci, U.; Gabrielli, C.; Huet, F.; Keddam, M. Noise resistance applied to corrosion measurements. I. Theoretical analysis. J. Electrochem. Soc. 1997, 144, 31-37. [CrossRef]

47. Bacon, R.C.; Smith, J.J.; Rugg, F.M. Electrolytic resistance in evaluating protective merit of coatings on metals. Ind. Eng. Chem. 1948, 40, 161-167. [CrossRef]

48. Mills, D.; Jamali, S.; Tobiszewski, M.T. Developing electrochemical measurements in order to assess anti-corrosive coatings more effectively. Prog. Org. Coat. 2012, 74, 385-390. [CrossRef]

49. Ohwaki, T.; Urushihara, W.; Kinugasa, J.; Noishiki, K. Aluminum Alloy Material and Plate Heat Exchanger with Superior Corrosion Resistance. US Patent 20100006277A1, 14 January 2010.

50. Sheffer, M.; Groysman, A.; Mandler, D. Electrodeposition of sol-gel films on al for corrosion protection. Corros. Sci. 2003, 45, 2893-2904. [CrossRef] 
51. Ma, W.; Wu, H.; Higaki, Y.; Otsuka, H.; Takahara, A. A “non-sticky" superhydrophobic surface prepared by self-assembly of fluoroalkyl phosphonic acid on a hierarchically micro/nanostructured alumina gel film. Chem. Commun. 2012, 48, 6824-6826. [CrossRef] [PubMed]

52. Park, Y.; Han, M.; Ahn, Y. Fabrication of superhydrophobic metal surfaces with self-assembled monolayers of silane derivatives having inter-hydrogen bonding. Bull. Korean Chem. Soc. 2011, 32, 1091-1094. [CrossRef]

53. Van, T.N.; Lee, Y.K.; Lee, J.; Park, J.Y. Tuning hydrophobicity of $\mathrm{TiO}_{2}$ layers with silanization and self-assembled nanopatterning. Langmuir 2013, 29, 3054-3060. [CrossRef] [PubMed]

54. Akporiaye, D.; Stöcker, M. Solid-state n.m.r. and XRD study of the thermal stability of VPI-5: Assignment of 31P and 27Al MAS n.m.r. spectra. Zeolites 1992, 12, 351-359. [CrossRef]

55. Gao, W.; Dickinson, L.; Grozinger, C.; Morin, F.G.; Reven, L. Self-assembled monolayers of alkylphosphonic acids on metal oxides. Langmuir 1996, 12, 6429-6435. [CrossRef]

56. Gao, W.; Reven, L. Solid-state nmr studies of self-assembled monolayers. Langmuir 1995, 11, $1860-1863$. [CrossRef]

57. McNatt, J.S.; Morgan, J.M.; Farkas, N.; Ramsier, R.D.; Young, T.L.; Rapp-Cross, J.; Espe, M.P.; Robinson, T.R.; Nelson, L.Y. Sonication assisted growth of fluorophosphate films on alumina surfaces. Langmuir 2003, 19, 1148-1153. [CrossRef]

58. Neff, G.A.; Page, C.J.; Meintjes, E.; Tsuda, T.; Pilgrim, W.C.; Roberts, N.; Warren, W.W. Hydrolysis of surface-bound phosphonate esters for the self-assembly of multilayer films: Use of solid state magic angle spinning 31p nmr as a probe of reactions on surfaces. Langmuir 1996, 12, 238-242. [CrossRef]

59. Phung, L.H.; Kleinert, H.; Jansen, I.; Häßler, R.; Jähne, E. Improvement in strength of the aluminium/epoxy bonding joint by modification of the interphase. Macromol. Symp. 2004, 210, 349-358. [CrossRef]

60. Lin, C.T.; Lin, P.; Hsiao, M.W.; Meldrum, D.A.; Martin, F.L. Chemistry of a single-step phosphate/paint system. Ind. Eng. Chem. Res. 1992, 31, 424-430. [CrossRef]

61. Gan, S.; Solimeno, R.D.; Jones, F.N.; Hill, L.W. Recent studies of the curing of polyester-melamine enamels. Possible causes of overbake softening. Proceedings of Water-Borne and Higher-Solids Coatings Symposium, Hattiesburg, MS, USA, 1-3 February 1989; pp. 87-108.

(C) 2017 by the authors. Licensee MDPI, Basel, Switzerland. This article is an open access article distributed under the terms and conditions of the Creative Commons Attribution (CC BY) license (http:/ / creativecommons.org/licenses/by/4.0/). 